\title{
OPTIMAL ANESTHESIA FOR ENDOSCOPIC PLACENTAL SURGERY
}

\author{
Sharon Davies* MD FRCPC \\ Jennifer Porter* MD \\ Craig E Pennell** MD PhD FRANZCOG \\ Farwaz Alkazaleh** MD \\ Greg Ryan** MB FRCSC FRCOG
}

Departments of Anaesthesia* and Obstetrics**, Mount Sinai Hospital, University of Toronto, 600 University Avenue, Toronto M5G 1X5, Canada.

\section{INTRODUCTION}

This retrospective study was designed to evaluate 3 anesthesia techniques - general anesthesia (GA), epidural anesthesia (ED) and conscious sedation (CS) using remifentanil and propofol that were employed for the first 8 0cases of fetoscopic laser ablation of placental vascular anastomoses for severe twin-twin transfusion syndrome (TTTS). There are no existing anesthetic practice guidelines in the literature for this procedure.

\section{METHODS}

Following hospital ethics board approval, detailed anesthetic, obstetric and procedure related data were obtained from the clinical records. Major and minor maternal complications and fetal outcomes were compared for the three techniques. Univariate comparisons were made using oneway ANOVA and Chi-square (Fisher's exact) tests. Multivariate logistic regression was used to identify independent associations with major maternal adverse outcomes.

\section{RESULTS}

Eighteen cases were performed under GA, 4 under ED and 58 under CS. There was no difference in maternal demographic data between the groups. The GA group recorded lower minimum systolic pressures $(\mathrm{p}=0.02)$, increased requirements for pressor therapy compared to CS (GA 44\%, ED 50\%, CS 4\%: p<0.01) and increased mean intraoperative intravenous fluids (GA 2650ml, ED 1667ml, CS 1007ml). Major maternal complications ( $\mathrm{n}=13$ : pulmonary edema, "mirror" syndrome, abruption, ICU admission and blood transfusion) were more common with general anesthesia as compared to the other groups $(\mathrm{p}<0.01)$. GA remained independently associated with major maternal complications in multivariate analysis (adjusted OR 36; $\mathrm{p}=0.03$ ) after controlling for intravenous fluid volume $(\mathrm{p}=0.01)$, pressor therapy $(\mathrm{p}=0.21)$, case number $(\mathrm{p}=0.43)$, severity of TTTS $(\mathrm{p}=0.35)$ and anesthetic time $(\mathrm{p}=0.10)$. Pulmonary edema within 24 hours of the procedure occurred in 8 women in this series, 7 of whom underwent GA. After controlling for covariates, total intravenous fluid volume was the only factor independently associated with the development of pulmonary edema (adjusted OR 4.5 for each $1000 \mathrm{~mL}$ increase in IV fluid).

\section{DISCUSSION}

GA for this procedure was associated with increased risk of major maternal complications, particularly pulmonary edema, when compared to ED and CS. The mechanism responsible for this increased risk is unknown and appears to be independent of procedure time, severity of TTTS, maternal arterial pressure, intravenous fluid volumes and the requirement for pressor agents. 